\title{
Taking unknown risks based on positive and negative information
}

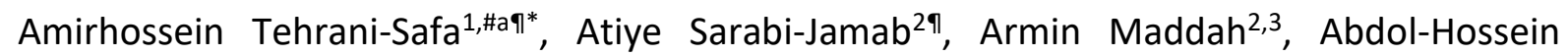
Vahabie $^{4,5}$, Babak Nadjar Araabi ${ }^{1}$, and Bahador Bahrami ${ }^{6,7^{*}}$.

${ }^{1}$ Control and Intelligent Processing Centre of Excellence, School of Electrical and Computer Engineering, College of Engineering, University of Tehran, P.O. Box 14395-515, Tehran, Iran;

2 School of Cognitive Sciences, Institute for Research in Fundamental Sciences (IPM), P.O. Box 19395-5746, Tehran, Iran;

${ }^{3}$ Biological Psychology of Decision Making, Institute of Experimental Psychology, Heinrich Heine University Düsseldorf, Düsseldorf, Germany;

${ }^{4}$ Cognitive Systems Laboratory, Control and Intelligent Processing Center of Excellence (CIPCE), School of Electrical and Computer Engineering, College of Engineering, University of Tehran, Tehran, Iran

${ }^{5}$ Department of Psychology, Faculty of Psychology and Education, University of Tehran, Tehran, Iran

${ }^{6}$ Faculty of Psychology and Educational Sciences, Ludwig Maximilian University, Munich, Germany;

7 Centre for Adaptive Rationality, Max Planck Institute for Human Development, Berlin, Germany.

\#a School of Cognitive Sciences, Institute for Research in Fundamental Sciences (IPM), P.O. Box 19395-5746, Tehran, Iran;

* Corresponding authors

E-mail: ahtehranisafa@gmail.com (AHTS), bbahrami@gmail.com (BB),

" These authors contributed equally to this work. 


\begin{abstract}
Many decisions have to be made under partial ambiguity where information is not available about the full probability distribution of risks. To decide in a principled way, one would have to make some assumption(s) about hidden risks. We examined how people may balance between the valence of the available information and the potential information concealed by the ambiguity.-Under partial ambiguity, people showed flexible skepticism towards the valence of the partially observable probabilistic information. When ambiguity size was small, risk taking was sensitive to valence: if the information was promising, ambiguity aversion increased, skeptically balancing the promising prospects of available positive evidence against the hazards of what might be hidden from the view. Conversely, when the available information was disappointing, ambiguity tolerance increased, cautiously anticipating more than what the available information promised. This flexible skepticism was not a trivially reflexive response to valence: when ambiguity was large (i.e., available information was unreliable), the valence of available information did not impact risk attitudes.
\end{abstract}




\section{Introduction}

When making decisions under uncertainty, knowing the probabilities of different outcomes simplifies thinking about how people may approach choice problems by allowing us to apply the principles of rational decision theory [1]. This family of theories give us clear guidelines about how one should decide and thereby enable straightforward hypotheses for what goes on in the decision makers' minds.

Under ambiguity, however, decision makers cannot calculate risk. This introduces important difficulties in understanding how people make decisions with incomplete information, which incidentally happens to be the case with most everyday life decisions. As an example toy model, take Ellsberg's famous demonstration: in a one-shot gamble to make a choice between a risky urn of 50 red (good) and 50 blue (bad) tokens and another, ambiguous urn of 100 tokens with unknown red/blue proportion, people tend to prefer choosing the former (risky) option over the latter (ambiguous) one [2]. Such "ambiguity aversion" may be interpreted to mean that individuals believe that the number of winning tokens in the ambiguous gamble must be fewer than in the risky one. This notion of subjective belief - called ambiguity attitude - about the likely structure of one's ignorance could help us understand how the agent may fill out the missing bit of information necessary to make a choice.

In many real-life situations, ambiguity does not necessarily turn up as the complete absence of all information but it also can indicate partially missing information. In such cases, one has to make up their mind with whatever partial information they have. "Partial" ambiguity attitude has been recently studied [3] [4] [5] by manipulating the relative size of the ambiguity while keeping the valence of the information neutral. Ambiguity aversion is also observed in the face of partial ambiguity. Examining ambiguity aversion under partial ambiguity raises important and new questions.

Available information often has some valence, sometimes promising benefit and other times cautioning against loss pushing us towards or away from embracing the ambiguity vs risk. In one study [6] employing theoretical methods and behavioral experiments asymmetric effects of positive and negative news were found. When available information supported a favorable outcome, ambiguity tolerance increased. However, unfavorable information did not affect ambiguity attitude. Similar asymmetric treatments of positive and negative cues for decision making under risk have been widely interpreted as the underlying cognitive basis of optimism bias [7] [8].

By employing an experimental paradigm that combined risky and ambiguous decision making, here we examined how subjective probability may be constructed from positive vs. negative partial 
information as the participants chose between a risky option vs another, partially ambiguous option. We quantified ambiguity attitude in humans by comparing preferences between varying risky and partially ambiguous gambles. Each trial of our experiment presented a choice between playing a risky or a partially ambiguous gamble (Fig $1 \mathrm{~A})$ with the same payoff size. We systematically and orthogonally manipulated (Fig 1B) the proportion of ambiguity/information as well as the valence of information by changing the proportion of good/bad news (i.e. positive vs zero reward). By applying a staircase method (Fig 1C) borrowed from sensory psychophysics, we estimated the risky equivalent of each partially ambiguous gamble. This equivalent risky gamble allowed us to infer each participant's subjective fractionation of ambiguity (Fig 1D).

Following from the earlier works on optimism bias under risk [8] [9], we predicted that greater ambiguity tolerance should be observed when available information has positive vs negative valence. Our results, however, demonstrated a much more nuanced behavior indicative of a flexible form of skepticism: when ambiguity size was tractable, subjective belief was sensitive to valence of information: if the information was promising, ambiguity aversion increased, skeptically balancing the promising prospects of available evidence against the hazards of what might be hidden from the view. Conversely, when the information was disappointing, ambiguity tolerance increased, cautiously encouraging the participant to be more adventurous than what the available information guaranteed. When ambiguity was large, ambiguity attitudes were not affected by valence of information. 


\section{Methods}

Participants: A total of 77 healthy participants (mean age $=27.4, S D=4.3$ ) were recruited in the study consisting of 36 females (19-37 y old) and 41 males (20-35 y old). Participants were from a wide range of academic disciplines and they were either at graduate level or in the last semester of their first degree. All participants signed an informed consent. Participants received monetary payment based on their decisions at the end of the experiment (see monetary payment).

Procedures: Participants were individually assessed for attitudes towards ambiguity. Each participant was briefed about the "game" and payment scheme. Participants knew that there is no "right answer" to any of the choices that they will face and they were only required to report their preferences. They were informed that some of their decisions would be randomly used to calculate their monetary reward, hence their choices would not result in any loss. Each participant had played a training session before the experiment to become familiar with the task procedures.

Main Task: Our experiment consisted of 270 two-alternative forced choice (2-AFC) trials that presented a choice between playing a risky or an ambiguous gamble with the same payoff. Two gambles were presented simultaneously on the computer screen in the form of pie charts which indicated the number of different tokens in each virtual urn and both urns contained 100 tokens (Fig 1A). The red and blue areas of the pie charts represented the ratio of red and blue tokens. Participants were told that they would "win" if a red token was drawn from their chosen virtual urn. The known proportion of tokens was also shown numerically. Pie charts were rotated randomly to avoid using visual alignment in decision between gambles.

To introduce ambiguity, a portion of one pie chart was blocked by gray. Participants were informed that each ambiguous gamble had an actual underlying winning ratio assigned to it which was hidden from the participant by the gray section. In this way, calculating the expected value of the ambiguous gamble was impossible. Participants were assured that a priori winning ratios were fixed during the experiment and would not be changed by experimenters.

We systematically varied the properties of ambiguous gambles across trials (Fig 1B). We crossed three Ambiguity Sizes, AS (25\%, 50\%, and $75 \%)$ with three ratios of winning tokens over total number of tokens in the known part which we call Known Winning Ratio, $\operatorname{KWR}(0.2,0.5$, and 0.8$)$. Different values of KWR imply different probabilities of winning to participants. For example, KWR $=0.2$ specified that in the known part of the urn $20 \%$ of tokens were winning tokens and $80 \%$ were null, so the delivered information is asymmetric in favor of losing the gamble. Following the same rationale, KWR $=0.5$ implied equal probability of winning vs not winning. Finally, $K W R=0.8$ meant that the information available to the participant favored winning. 
The experiment was conducted in 3 sessions with 30 blocks of 3 trials. In each session, a fixed KWR was employed. Within each block, 3 ambiguous gambles of different ambiguity sizes were proposed in randomly interleaved order. The order of which KWR to display in which session were randomized across participants. Participants had unlimited time to respond and they did not receive feedback for trials.

To estimate the equivalent risky gamble corresponding to each ambiguous gamble, we employed an approach similar to staircase method with variable step-size which is commonly used in psychophysics studies [10]. The winning ratio of the risky gamble was adjusted adaptively across the session by a stochastic approximation staircase [11]. If the participant preferred the risky gamble over the ambiguous one, then winning ratio of the risky gamble was decreased in the next corresponding trial; if he/she preferred the ambiguous gamble then winning ratio of the risky gamble was increased. The changes in the winning ratio of the risky gamble were restricted to the AS. Thus, as the winning ratio of the risky gamble was changed depending on participant's choice, the subjective fractionation of ambiguous part was estimated as the staircase covered the Ambiguity Size.

The staircase started with proposing the winning ratio of risky gamble equal to number of winning tokens in the ambiguous gamble plus half of the AS. The initial step-size of staircase was equal to a third of AS and decreased as the participant reversed his/her choice. Decrement of the step size followed a harmonic series (i.e. AS/4, AS/5, ..., AS/10) and remained constant when it reached AS/10 (Fig 1C). The choice of large initial step-size and its progressive decrement guaranteed the convergence of staircase to the Point of Subjective Ambivalence (PSA) [12]. The minimum winning ratio proposed by the staircase was equal to number of winning tokens in the known part of the ambiguous gamble, while the maximum winning ratio proposed to participants was given by fractioning all of the ambiguous part as winning tokens.

After the experiment, the participants completed the Revised Life Orientation Test (LOT-R) [13]. We used this questionnaire as a measure of trait optimism/pessimism and its results did not affect the participant's payment.

Monetary Payment: People might not perform realistically for hypothetical situations [14], hence we informed participants that we would randomly select one trial from each session ( 3 trials in total) to run the selected gamble in that trial for their monetary payment at the end of the experiment. We labeled numbers from 1 to 100 with red/blue colors with respect to the proportion of tokens of the chosen gamble in that trial. We asked participants to pick a number between 1 to 100 . If the color assigned to the number was "red" then we paid them 100K Rials (equivalent to $3 \$$ ). Each participant also received $100 \mathrm{~K}$ Rials for participating in the experiment. 
Simulating agent to compare the experimental behavior with a number of possible alternative strategies: We anticipated that there might be a range of strategies to explain the ambiguity-resolving behavior. We simulated 1000 agents for each of our suggested strategies. The probability that the agent chooses an ambiguous gamble is calculated by a single logistic function; $P_{\text {choice }}=e^{\beta \cdot p 2} /\left(e^{\beta . p 1}+e^{\beta . p 2}\right)$, where $p_{1}$ is the probability of winning in risky gamble, and $p_{2}$ is the subjective probability of winning in ambiguous gamble. $\beta$ is the slope of the logistic function, or a noise parameter. For $\beta$ near zero, choosing the ambiguous gamble or risky gamble have nearly the same probability and for high noise parameter, the probability of the choosing ambiguous gamble tend to 1 . We used $\beta=0.2$ for simulating random selection. Based on staircase results, we derived the corresponding AA in all 9 experimental conditions for each simulated agent. 


\section{Results}

The risky gamble consisted of winning (red) and null (blue) tokens where winning resulted in $100 \mathrm{~K}$ Rials payoff. The ambiguous gamble was similar to the risky gamble but a portion of tokens were not disclosed to participants and they did not know the ratio of winning and null tokens in this unclosed proportion. Gambles were not played until the end of the experiment. Participants reported their preference between one ambiguous and one risky gamble at each trial. No feedback was given about gamble outcomes during the experiment. Fig $1 \mathrm{~A}$ depicts a sample trial consisting of a risky gamble (left pie chart) with fully known probabilities of outcomes: $40 \%$ chance of winning and $60 \%$ chance of getting nothing. The right pie chart depicts a symmetric ambiguous gamble with partially known probabilities of outcomes $(25 \%<$ chance of winning $<75 \%)$.

The winning ratio of the risky gamble varied systematically across trials, to determine how the Known Winning Ratio (KWR) and Ambiguity Size (AS) influenced participant's choice. AS is the fraction of the ambiguous gamble covered by the grey sector. The experimental design combined three levels for AS (Ambiguity Size: small (25\%), medium (50\%), large (75\%)), with three values for KWR (Known Winning Ratios: negative valence of information (0.2), neutral (0.5), positive valence of information (0.8)) giving rise to 9 conditions. Fig $1 \mathrm{~B}$ shows the 9 conditions resulting from the $3 \times 3$ design.

Subjective attitudes towards ambiguity were elicited using a staircase technique with variable stepsize. Exemplary, a run of the staircase for a designated ambiguous gamble $(A)$ is shown in Fig $1 C$. Every time the participant chooses the risky gamble $(R)$, in the next step the staircase proposes a risky gamble with an increased number of null tokens. Every time the participant chooses the ambiguous gamble then the staircase updates the risky gamble with an increased number of winning tokens.

We defined the Point of Subjective Ambivalence (PSA) between ambiguous and risky gambles as the average of the last 15 risky winning ratios proposed to the participant within a run of 30 trials. We used the PSA to infer how the participant must have fractionated the ambiguity into win $\left(n_{s}\{W\}\right)$ and null $\left(n_{s}\{N\}\right)$ subcomponents (Fig 1D). We then calculated the Ambiguity Attitude (AA) for each participant in each condition by:

$$
A A=\frac{n_{s}\{W\}}{n_{s}\{W\}+n_{s}\{N\}}
$$

AA is a number between 0 and 1 . A value of 0.5 shows that the participant split the ambiguous part equally between winning and null tokens (Ambiguity Neutrality). Values higher than 0.5 indicate that the participant divided the ambiguity in favor of the winning tokens (Ambiguity Seeking, Fig 1D right pie chart). Values lower than 0.5 show that the participant interpreted the ambiguity negatively favoring null tokens (Ambiguity Aversion). 
A

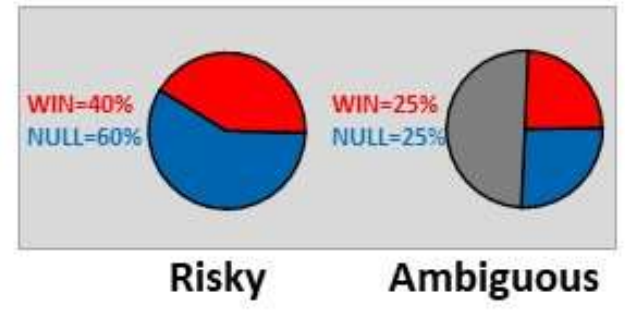

C

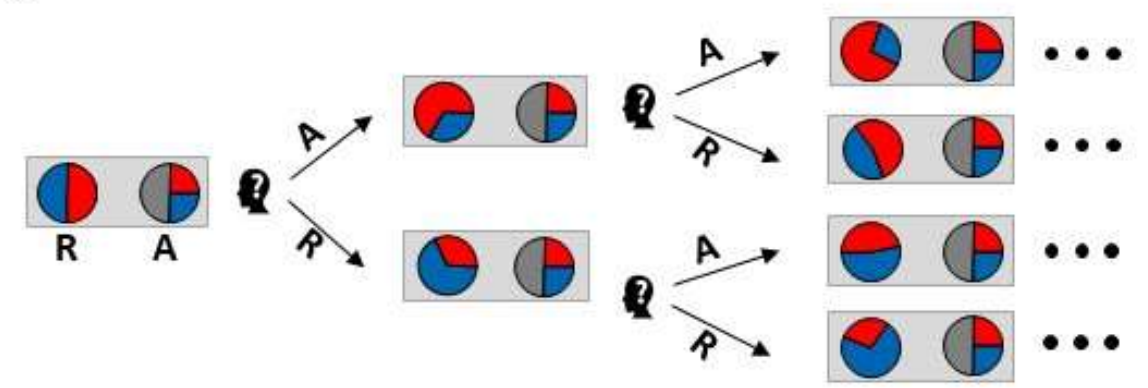

D
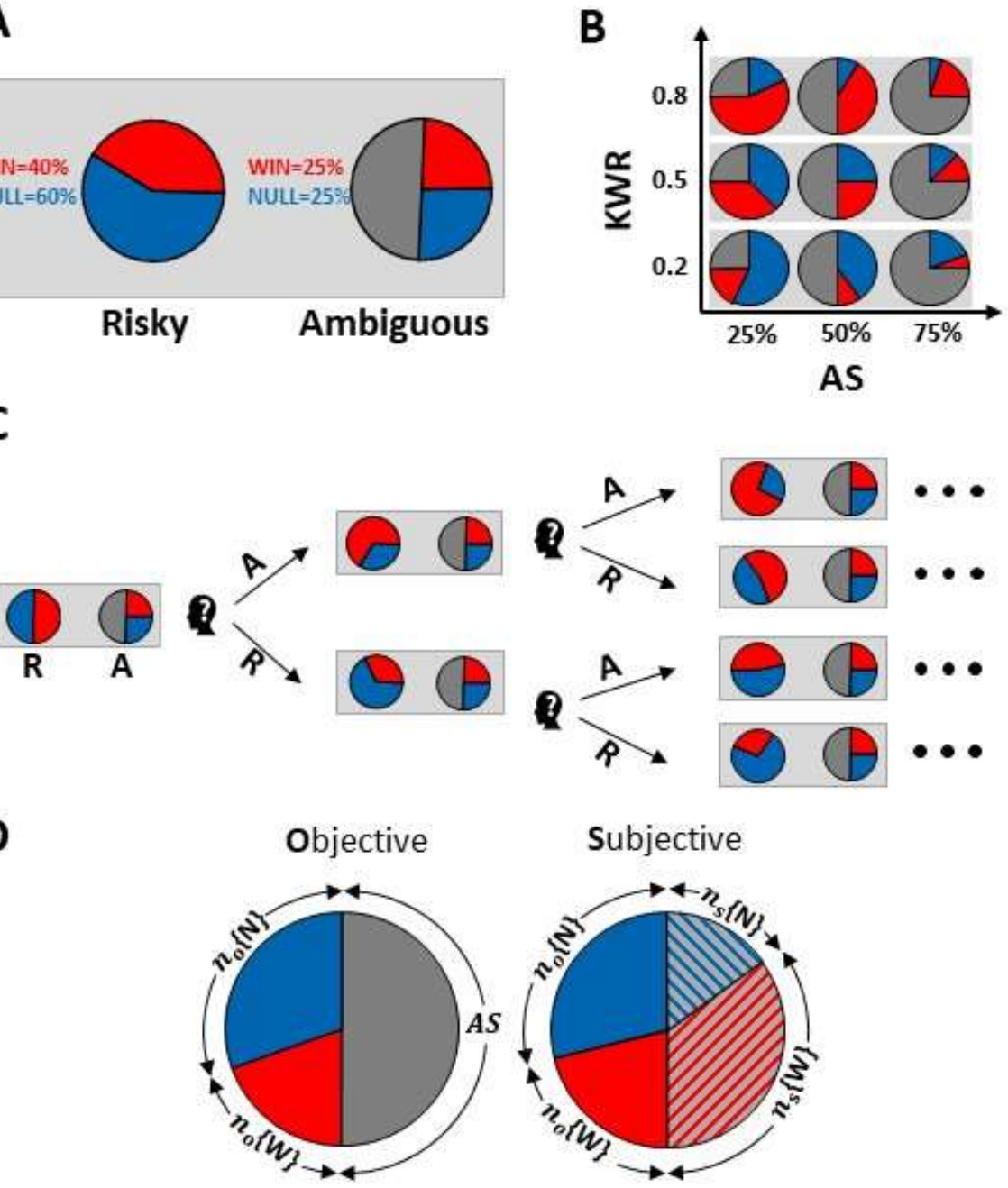


\section{Fig 1. Stimuli and Experimental design.}

(A) The pie chart on left side represented the risky gamble and the one on right represented the ambiguous gamble. Proportion of tokens in known part of both gambles was shown numerically next to the corresponding pie chart. (B) All ambiguous gambles designed for the experiment. On the horizontal axis, the size of hidden part increases from left to right. On the vertical axis, the number of winning tokens in known part increases from down to up. KWR: Known Winning Ratio, AS: Ambiguity Size (C) Tree representation of staircase for one of the ambiguous gambles in one session. The winning ratio of risky gamble was changed in next block of experiment depending on participant's choice. By choosing the risky (ambiguous) gamble staircase increases the proportion of null (winning) tokens in risky urn on next trial. A: Ambiguous gamble, R: risky gamble. (D) Example for fractionation of ambiguous gamble by participant. $n_{0}\{$.$\} indicates the number of tokens$ in known part of ambiguous gamble and $n_{s}$ L. $\}$ relates to subjective fractionation of ambiguous part. AS: Ambiguity Size, W: Wining tokens, N: Null tokens.

By employing various KWRs (Fig 1B), we offered negative/neutral/positive valence of information to the participants for measuring the effect of the valence of the information on ambiguity attitude. Previously, ambiguity attitude has been studied only under neutral information, where the probability of winning and not-winning represented by partial information was equal [3] [4] [5]. Our work goes beyond those previous studies by introducing different valence of information. We tested our hypothesis about the impact of the valence of information on the relative likelihood that participants attach to the ambiguous part. We predicted that the ambiguity attitude would be greater in positive valence than the negative valence of information (Fig $2 \mathrm{~A}$ ).

A 3-way mixed ANOVA (KWR: negative, neutral and positive, AS: small, medium and large, Gender: male and female, AA: dependent variable) was employed showing a significant main effect of KWR $[F(2,679)=12.18, p=6.3 e-6]$. There was also a significant effect of gender $[F(1,679)=7.24, p=0.01]$, but no main effect of ambiguity size $[F[2,679]=0.14, p=0.87]$ and no significant interaction between the independent variables (S1 Table).

The main effect of KWR on AA revealed a marked asymmetry about resolving ambiguity in different conditions. For each participant, we calculated the average of AAs at a fixed KWR. A comparison between $A A$ in positive vs. negative conditions ( $K W R=0.2$ vs. 0.8 ) confirmed that the $A A$ in positive condition was significantly less than the $A A$ in negative condition (paired t-test; $t[76]=3.43, p=0.001$ )

(Fig 2B). People were less ambiguity tolerant in positive condition relative to negative condition. The ambiguity tolerance decreased as the information was more favorable. We concluded that dividing the structure of ignorance was biased respect to given evidence. People assume that the structure of hidden part would be different with the structure of available evidence and fill out the missing bit of information differently when deal with ambiguity. This kind of pessimism about given information in the domain of ambiguity, need more explanation and analysis. 
Additional analysis showed that on average female participants were more ambiguity averse than males (S1 Fig, S1 Table). The lack of a main effect of AS on AA indicated that the size of ambiguity had not affected the Ambiguity Attitude. This was consistent with previous studies on decision making under partial ambiguity [15] [16].

To explain how subjective structure of probability distribution in hidden part was biased with the accessible information in the known part, we defined the Optimism Index (OI) by:

$$
\boldsymbol{O I}(\text { Optimism Index })=\frac{\Delta A A}{\Delta K W R}=\frac{A A_{K W R_{2}}-A A_{K W R_{1}}}{K W R_{2}-K W R_{1}}
$$

A

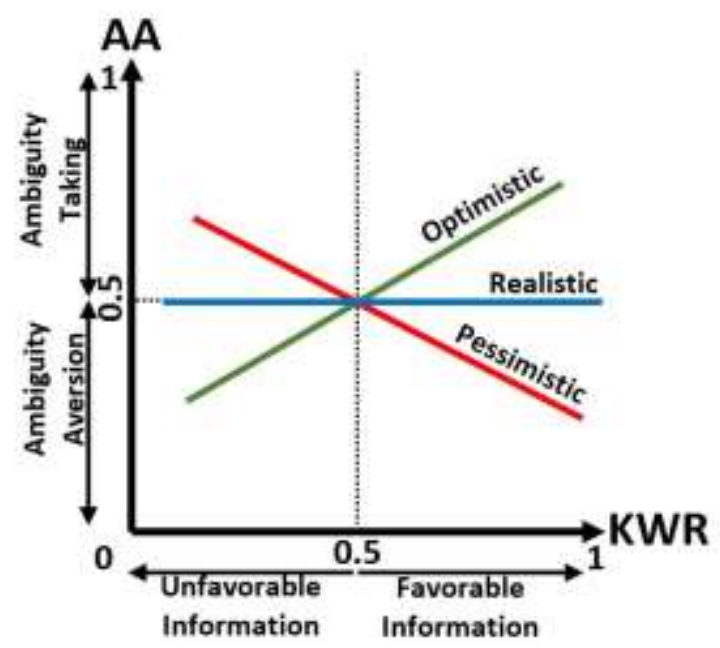

B

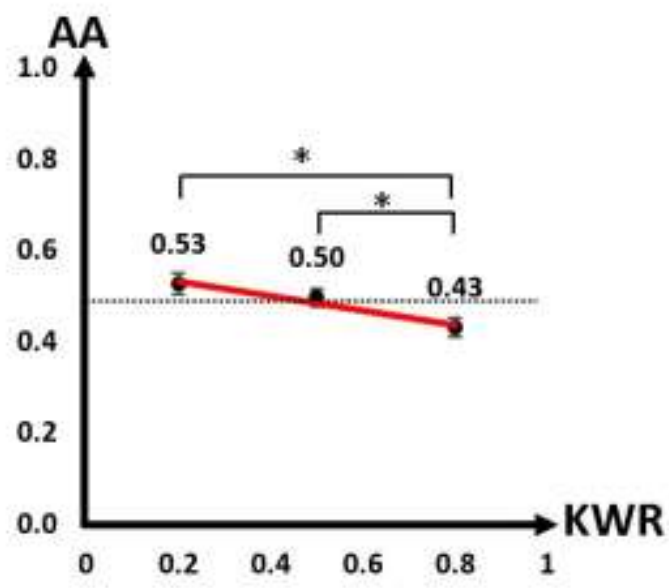




\section{C}

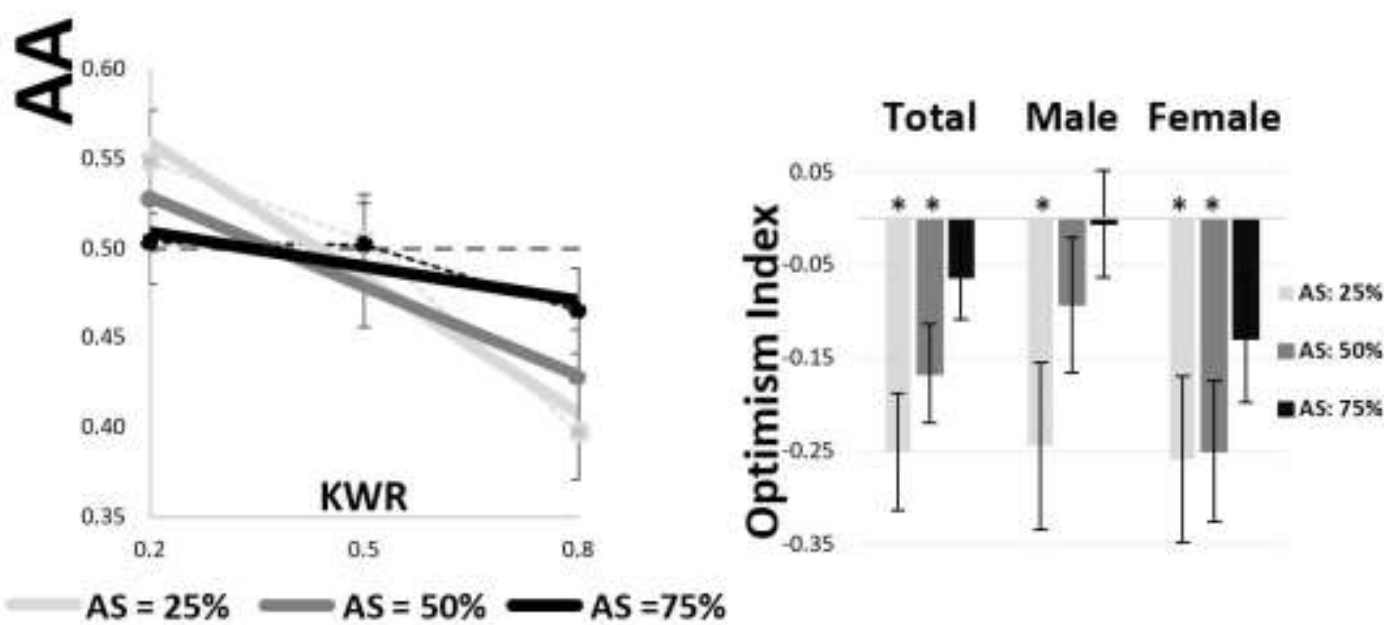

Fig 2. Ambiguity attitude and Optimism Index in different conditions.

(A) Schematic representation of the valence-dependent modifying account for our experiment. For an optimistic person the higher KWR results in the higher ambiguity attitude (positive slope). A pessimistic person has negative slope. A realist keeps the same AA irrespective of the valence of information (zero slope) (B) For a single participant we calculated the average of AAs at a fixed KWR. The mean and SEM of averaged values have been illustrated. Pooled over all participants. * defines significant difference of mean in two-sample paired t-test $(p<0.05)$. AA: Ambiguity Attitude, KWR: Known Winning Ratio (C) A regression line was fitted for each participant. Then mean of regression lines is illustrated for each fixed AS pooled over total dataset (D) Mean and SEM of Optimism Indexes illustrated for each AS and gender, * defines $p<0.05$.

Let explain how the novel optimism Index works. An optimist person has positive optimism index. It means that she gets more ambiguity tolerance (less ambiguity averse) in favorable condition than unfavorable condition. Therefore, for an optimistic person the higher KWR results in the higher ambiguity attitude (positive Optimism Index). Inversely, a pessimist person has negative Optimism Index. Her ambiguity aversion in positive condition is bigger than in negative condition. If the information biased toward the winning, she assumes less winning tokens in the hidden part. A realistic person doesn't change her ambiguity attitude as a consequence of valence of information. In the other word, proposed information cannot change her subjective belief about the distribution of tokens in the hidden part. (Fig 2A, S2 Fig).

The novel introduced Optimism Index measures the sensitivity of people to the given information. We know that ambiguity attitude also traces some kind of optimism/pessimism trait. But we should clarify 
and emphasize that Optimism Index measures totally different issue. When we called someone ambiguity averse it means she generally dislikes ambiguous options and perceive ambiguity as an undesirable situation. But here Optimism Index, measures how she changes her subjective probability in line with accessible data. For example, if the biased structure to winning could lead to reduced ambiguity aversion. From this definition, we understand that someone could have a positive optimism Index and simultaneously she could be ambiguity averse.

To calculate the OI, for each participant we regressed AA on KWR values separately for each AS. Figure $2 \mathrm{C}$ shows the regression line for each Ambiguity Size pooled across all participants. Figure 2D shows the optimism index for each level of AS separately for male and female participants as well as for the entire dataset. In our empirical data, a two-way ANOVA (dependent variable: Optimism Index) with factors of AS and gender, showed that there was no main effect of gender $(F[1,225]=2.47, p=0.12)$ but a marginally significant main effect of $\operatorname{AS}(F[2,225]=2.92, p=0.056)$ on slopes. A comparison between $\mathrm{OI}$ in $\mathrm{AS}=75 \%$ with zero confirmed that there was no significant difference (one sample ttest with zero; $A S=75 \% ; t[76]=-1.42, p=0.16)$. When the ambiguity size was large, people tended to be more realistic. Moreover, the Ols in small and medium ambiguity size conditions were significantly less than zero (one sample t-test with zero; $A S=50 \%$ : $t[76]=-3.14, p=0.0024 ; A S=25 \%$ : $t[76]=-3.99, p=1.00 E-04)$ (S2 Table). When the ambiguity size was tractable, people tended to be pessimist.

Additional control measures showed that optimism indexes were not correlated with participants' trait optimism (LOT-R) (AS = 25\%: $r=0.03, p=0.81 ; A S=50 \%: r=0.1, p=0.39 ; A S=75 \%: r=0.18, p=$ 0.13) (S3 Fig).

As we will see below, the analysis of optimism index gives us straightforward handle to compare the empirical results to a number of plausible cognitively inspired hypotheses for the mental process shaping our subjects' decision making under ambiguity (Fig 3B). A key strength of this approach is that the predictions drawn from these seemingly similar hypotheses are radically different from one another once they are applied to the context of the experimental setup. As a result, even a qualitative comparison of the data (Fig 2C-D) to the predictions (Fig 3B-C) communicates our point sufficiently. 
A

Objective
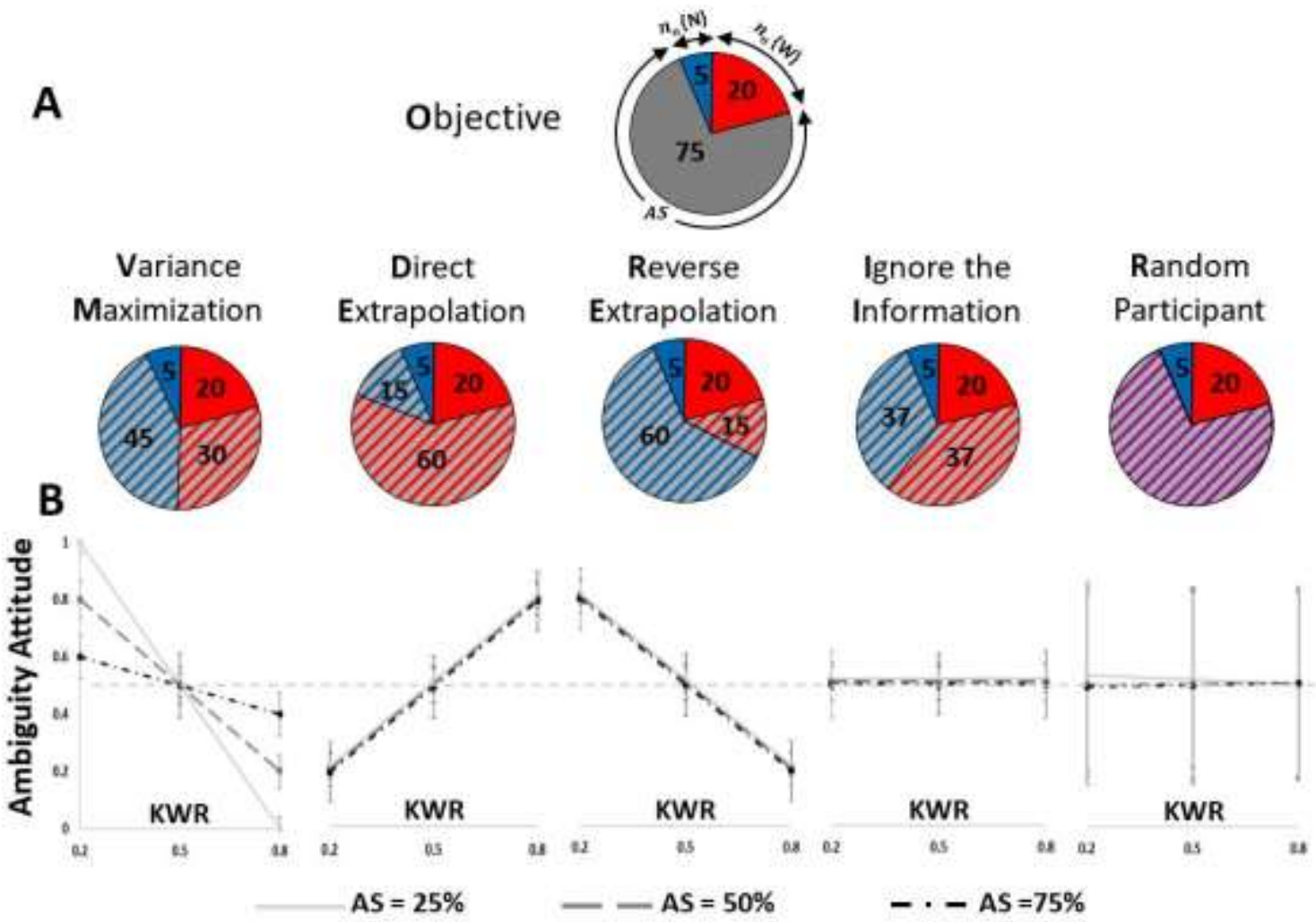

C

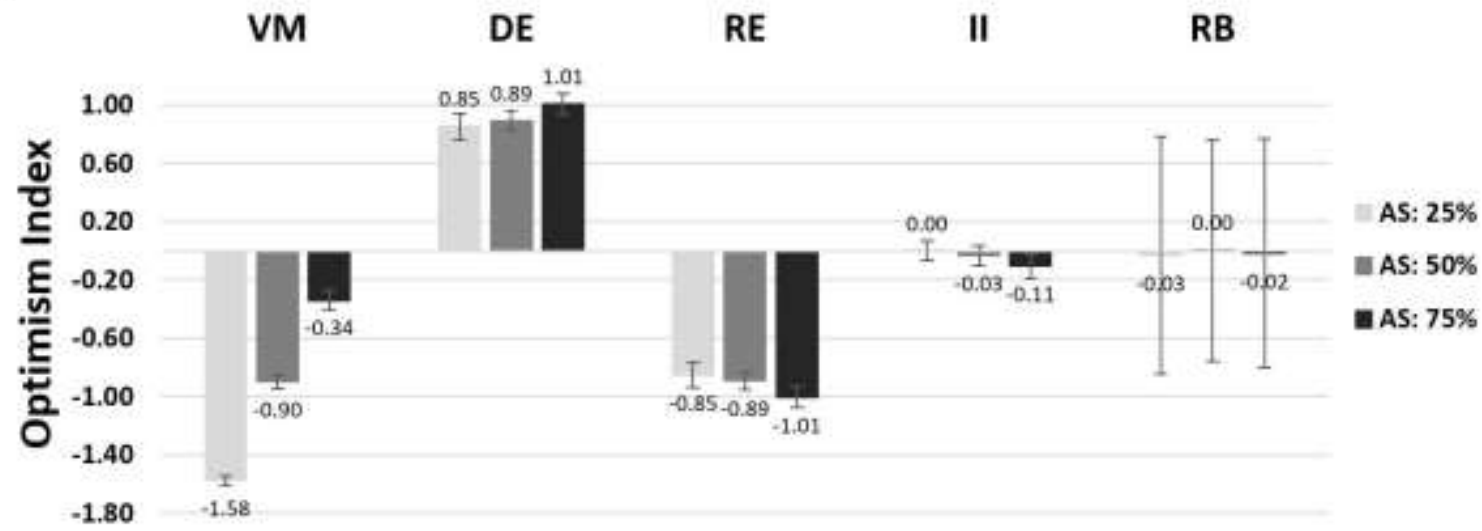

Fig 3. Different plausible strategies for resolving ambiguity and their model predictions in compare with empirical results.

(A) Quantitative calculation of resolving ambiguity with example for different strategies. $n_{o}\{$.$\} indicates$ the number of tokens in known part of ambiguous gamble and $n_{s}\{$.$\} relates to subjective fractionation$ of ambiguous part, AS: Ambiguity Size, W: Wining tokens, N: Null tokens. (B) Model Predictions for each strategy. For all strategies, to estimate the Optimism Indexes, we simulated 1000 agents for each 
strategy, and next for each agent we calculated values of AA. The mean and standard deviation of AA values have been illustrated. In these figures the $X$-axis corresponds to three steps of KWR and $Y$-axis corresponds to computed Ambiguity Attitude (AA). Each line indicates the relationship between KWR and AA (Optimism Index) in a fixed AS. At KWR $=0.5$ and with different ambiguity sizes, the values of $\mathrm{AA}$ are equal to 0.5 by following any of the considered strategies. (C) Mean and SEM of Optimism Indexes for alternative strategies illustrated for each AS.

Variance Maximization. If the participants interpreted every ambiguous gamble as a 50-50 risky urn, then the subjective ambivalence for each of the 9 conditions would indicate that $\left(n_{S}\{W\}+n_{O}\{W\}=n_{s}\{N\}+n_{O}\{N\}=50\right)$. This would be equivalent to assume maximum variance in the ambiguous gamble (Fig 3A). A participant following this extremely simple and intuitive strategy would compare the probability of winning in risky gamble with $50 \%$. Remarkably, such a simple strategy would correspond to a very elaborate pattern of different negative slopes for the relationship between KWR and AA for different ambiguity sizes (Fig 3B).

Direct Extrapolation. Participants may fractionate the ambiguous part in exactly the same proportion as the known part (Fig 3A). The choice would involve comparing the ratio of winning tokens in known part with the ratio of winning tokens in the corresponding risky gamble, where the quantitative outcome is $\left(n_{S}\{W\} / n_{S}\{N\}=n_{0}\{W\} / n_{O}\{N\}\right)$. This strategy predicts a unique positive slope or the relationship between AA and KWR (Fig 3B).

Reverse Extrapolation. A paranoiac participant may assume that the portion of tokens in ambiguous part is the inverse of the proportion displayed in the known sector (Fig 3A). The quantitative outcome is $\left(n_{s}\{W\} / n_{s}\{N\}=n_{0}\{N\} / n_{0}\{W\}\right)$ which corresponds to predicting the same fixed negative slope for all ambiguity sizes (Fig 3B).

Ignoring the information. Participants may not incorporate the partial information to resolve ambiguity, always splitting the ambiguous part in half. Participants following this strategy would not adjust their belief in response to variation of partial information. AA would be independent of KWR (Fig 3B) but for a fixed intercept indicating "Ambiguity Aversion" and "Ambiguity Seeking".

Random behavior. Finally, a useful null hypothesis is the one These predictions were obtained by simulating the agent that would take up each strategy (See Method) and calculating the simulated agent's AA (Fig 3B).

Thus, comparison of our experimental results (Fig 2D) with the predictions of the five strategies (Fig 3C) support the Variance Maximization strategy. 
A further hypothesis drawn from the Variance Maximization strategy is that if the available information is already consistent with maximum variance (i.e. $K W R=0.50$ ), then participant should have a much simpler task at hand requiring much less cognitive effort for disambiguation of the unavailable information. This would lead to the prediction that response times should be shorter when $K W R=0.50$ compared to when KWR $<>0.50$. On the other hand, many previous studies have shown that choice response times in human and non-human primates [17] [18] increase with variance in the evidence. These, previous studies would predict maximum response time in KWR $=0.50$.

We analyzed the Response Times (RT; Fig 4) of choices between risky and ambiguous gambles (RTs longer than 20 seconds were excluded from analysis). Using a one-way ANOVA on RTs indicated that there is a main effect of $\operatorname{KWR}(F[2,76]=2.73, p=0.05)$. There was a significant difference between conditions with negative/positive valence of information (KWR $=0.2$ and 0.8 ) and neutral condition $(K W R=0.5)($ paired t-test; $20 \%$ vs $50 \% ; t[76]=3.44, p=0.0009 ; 80 \%$ vs $50 \% ; t[76]=-3.14, p=0.002$, Fig 4).

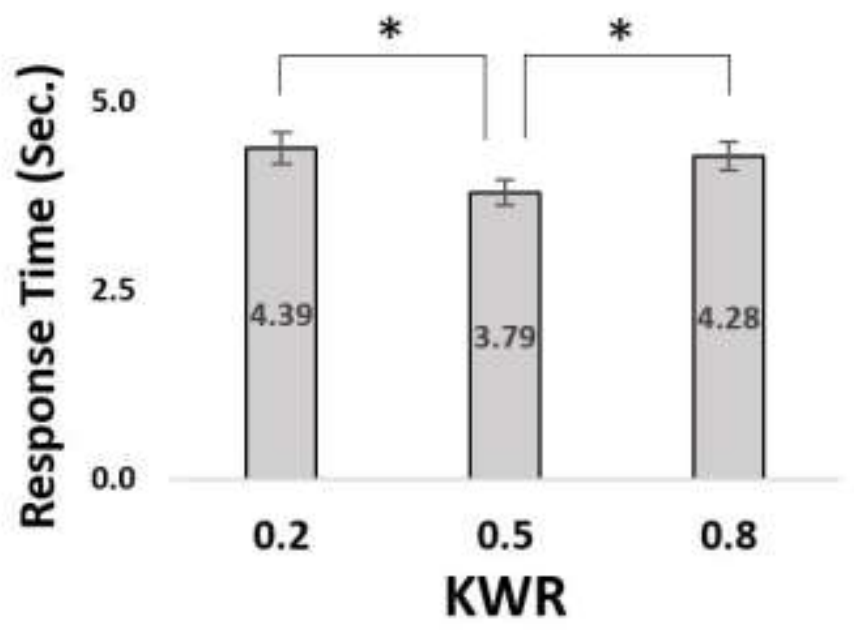

Fig 4. Comparing Response time in different Known Winning Ratio(KWR)

For a single participant we calculated the average of values of RT in 3 different ASs at a fixed KWR. The mean and SEM of averaged values have been illustrated. * indicates significant difference of means by two-sided paired t-test $(p<0.05)$. RT: Response Time, KWR: Known Winning Ratio. 


\section{Discussion}

Not much is known about the role of information in constructing subjective belief under ambiguity, where the probability distribution over uncertain events is partially or completely unknown. To address this question, our study focused on how individuals use the evidence they have to disambiguate what they do not know.

We combined a staircase procedure commonly used in sensory psychophysics [19] [10] with a classical risky choice paradigm in behavioral economics to estimate and extract the participants' ambiguity attitude. We directly elicited participants' ambiguity attitude by revealing their preferences in choosing between risky and ambiguous gambles in the context of an adaptive staircase. To test our main hypothesis, we introduced a novel approach by employing partial ambiguity which goes beyond previous studies of decision making under ambiguity [5] [20].

Some recent studies investigate how information could change ambiguity aversion. In [21], they learnt people about Ellsberg paradox and their own potentially suboptimal decisions in ambiguity domain. Results showed that this intervention reduced participant's ambiguity aversion. Generally showed that information could modify human suboptimal strategies in face of ambiguity.

In [6], By means of empirical and theoretical methods, they investigated how favorable and unfavorable information could alter value of ambiguous options. They showed that information added in favor of the winning raise the value of ambiguous gamble in the eyes of gambler. However, no effect was found when the information was in favor of losing the gamble. Their valuable work was distinct form our work in some aspects. First, they measured willingness to pay (WTP) balancing two factors: ambiguity aversion and subjective likelihood estimates, while we only focus on ambiguity aversion and how it could be swing by asymmetrical partial information. Second, most of their conditions were special cases that excluded from our task, for example $(0 \%, 25 \%)$ and $(50 \%, 0 \%)$ in their task which equals to $K W R=0$ and $K W R=$ infinity respectively.

Previous studies used a parametric computational model to interpret the ambiguity attitude, in most of cases a soft-max function has been employed to model probability of choosing the ambiguous gamble. Those previous works estimated the ambiguity attitude by applying a nonlinear optimization constrained by the participant's choice, which require numerous assumptions about the shape of distribution [5] [20]. In our study we fixed the monetary payment for both gambles and changed the winning ratio of risky gamble. Our non-parametric method based on staircase procedure empowered 
us to directly measure the ambiguity attitude. Therefore, we do not make any assumptions which may have affected the computational analyses.

Our results also showed a valence-dependent asymmetry in how people handle promising and disappointing information to decide about what they do not know. People do not fully trust the available evidence when they face ambiguity. Promising information pushes people to change their belief skeptically as they balance the promising prospects of available evidence against the hazards of what might be hidden from the view. Conversely, disappointing information fails to thwart people from being adventurous about what might be hidden versus what the evidence suggests.

In an unknown environment, people might have interpreted the evidence as deceptive effort, as if somebody might have wanted to lead them on to take a bad risk or lose some benefits [22] [23]. Our results are consistent with these previous reports of impact of context on role of valence in ambiguity domain.

Although there are a number of advantages to holding positive expectations, there seem to be obvious disadvantages to ignoring negative information such as underestimating risks. The asymmetric belief formation has been blamed for a host of disasters such as overly aggressive medical decisions [24], illpreparedness in the face of natural catastrophe [25] and financial collapse [26]. Moreover, positively biased views of the self can lead to error and cost, as shown for example in overconfident traders [27]. It seems that in unknown environment such a pessimism attitude could help us to handle information better when to decide about what we do not know.

A previous work concluded that the individuals with the greater ambiguity tolerance have more tendency to trust on other people during social decisions [28]. Although some previous studies in nonsocial domain illustrated that individuals with higher ambiguity tolerance are more optimistic about future according to LOT-R test [29], our new measure showed that the amount of optimism or pessimism about life is not related with the optimism in the ambiguity domain. Future studies will be needed to disentangle the relationship between personality and behavior in ambiguity domain. 


\section{Acknowledgement}

BB was supported by the European Research Council (ERC) under the European Union's Horizon 2020 research and innovation program (819040 - acronym: rid-O). BB was supported by the NOMIS foundation and the Humboldt Foundation.

\section{Author Contributions}

Supervision, Funding acquisition: BNA, BB

Conceived and designed the experiments: AHTS, AS, AHV, AM

Conducted the experiments: AHTS, AS

Analyzed the data and Developed the model: AHTS, AS

Wrote the paper: AHTS, AS, AM, BB

\section{References}

1. Kahneman D, Tversky A. Prospect Theory: An Analysis of Decision under Risk. Econometrica. 1979;47: 263-291.

2. Ellsberg D. Risk, ambiguity, and the Savage axioms. Q J Econ. 1961; 643-669.

3. Hsu M, Bhatt M, Adolphs R, Tranel D, Camerer CF. Neural Systems Responding to Degrees of Uncertainty in Human Decision-Making. Science (80- ). 2005;310: 1680-1683.

4. Huettel SA, Stowe CJ, Gordon EM, Warner BT, Platt ML. Neural Signatures of Economic Preferences for Risk and Ambiguity. Neuron. 2006;49: 765-775.

5. Levy I, Snell J, Nelson AJ, Rustichini A, Glimcher PW. Neural representation of subjective value under risk and ambiguity. J Neurophysiol. 2010;103: 1036-1047.

6. Peysakhovich A, Karmarkar UR. Asymmetric effects of favorable and unfavorable information on decision making under ambiguity. Manage Sci. 2016;62: 2163-2178.

doi:10.1287/mnsc.2015.2233

7. Lefebvre G, Lebreton M, Meyniel F, Bourgeois-Gironde S, Palminteri S. Behavioural and neural characterization of optimistic reinforcement learning. Nat Hum Behav. 2017;1: 1-9. doi:10.1038/s41562-017-0067

8. Sharot T. The optimism bias. Current Biology. Cell Press; 2011. pp. R941-R945. doi:10.1016/j.cub.2011.10.030

9. Sharot T, Garrett N. Forming Beliefs: Why Valence Matters. Trends in Cognitive Sciences. Elsevier Ltd; 2016. pp. 25-33. doi:10.1016/j.tics.2015.11.002

10. Garci'a-Pe'rez MA. A cautionary note on the use of the adaptive up - down method. J Acoust Soc Am. 2011;130: 2098-2107. 
11. Faes L, Ricci L, Vescovi M, Antolini R. Small-sample characterization of stochastic approximation staircases in forced-choice adaptive threshold estimation. Percept Psychophys. 2007;69: 254-262.

12. Scheier MF, Carver CS, Bridges MW. Distinguishing Optimism From Neuroticism (and Trait Anxiety, Self-Mastery, and Self-Esteem): A Reevaluation of the Life Orientation Test. J Pers Soc Psychol. 1994;67: 1063-1078.

13. Holt C a, Laury SK. Risk Aversion and Incentive Effects. Am Econ Rev. 2002;92: 1644-1655. doi:10.1257/000282802762024700

14. Dimmock SG, Kouwenberg R, Wakker PP. Ambiguity Attitudes in a Large Representative Sample. Manage Sci. 2016;62: 1363-1380.

15. Inukai K, Takahashi T. Decision under ambiguity: Effects of sign and magnitude. Int J Neurosci. 2009;119: 1170-1178.

16. Lopez-Paniagua $D$, Seger $C$ a. Coding of level of ambiguity within neural systems mediating choice. Front Neurosci. 2013;7: 1-19. doi:10.3389/fnins.2013.00229

17. Zylberberg A, Fetsch CR, Shadlen MN. The influence of evidence volatility on choice, reaction time and confidence in a perceptual decision. Elife. 2016;5: 1-31. doi:10.7554/eLife.17688

18. Kiani R, Shadlen MN. Representation of confidence associated with a decision by neurons in the parietal cortex. Science. 2009;324: 759-64. doi:10.1126/science.1169405

19. Treutwein B. Adaptive Psychophysical Procedures. Vision Res. 1995;35: 2503-2522.

20. Tymula A, Rosenberg LA, Roy AK, Ruderman L, Manson K, Glimcher PW. Adolescents' risktaking behavior is driven by tolerance to ambiguity. Proc Natl Acad Sci. 2012; 1-6.

21. Jia R, Furlong E, Gao S, Santos LR, Levy I. Learning about the Ellsberg Paradox reduces, but does not abolish, ambiguity aversion. Aimone JA, editor. PLoS One. 2020;15: e0228782. doi:10.1371/journal.pone.0228782

22. Shepperd J a, Findley-Klein C, Kwavnick KD, Walker D, Perez S. Bracing for loss. J Pers Soc Psychol. 2000;78: 620-34.

23. Sweeny K, Krizan Z. Sobering up: A quantitative review of temporal declines in expectations. Psychol Bull. 2013;139: 702-724.

24. Paling J. Strategies to help patients understand risks. BMJ. 2003;327: 745-748. doi:10.1136/bmj.327.7417.745

25. Paton D. Disaster preparedness: A social-cognitive perspective. Disaster Prev Manag An Int J. 2003;12: 210-216. doi:10.1108/09653560310480686

26. Shefrin H. How Psychological Pitfalls Generated the Global Financial Crisis. (Rochester, NY: Social Science Research Network). 2009. doi:10.2139/ssrn.1523931

27. Barber BM, Odean T. The Courage of Misguided Convictions : The Trading Behavior of Individual Investors. Finant Anal J. 1999;55: 41-55.

28. Vives ML, Feldmanhall O. Tolerance to ambiguous uncertainty predicts prosocial behavior. Nat Commun. 2018;9: 25-27. doi:10.1111/j.1474-919X.1989.tb02761.x

29. Pulford BD. Is luck on my side? Optimism, pessimism, and ambiguity aversion. Q J Exp Psychol. 2009;62: 1079-1087. 


\section{Supporting information}

S1 Fig. The mean and Standard Error of Mean (SEM) for Ambiguity Attitude (AA) across all conditions separated for males and females

S2 Fig. univariate plot for Optimism Index separated by genders

S3 Fig. The extracted measures of LOT-R questionnaire vs. Ol separated by gender

S1 Table. Results of 3-way ANOVA analysis on Ambiguity Attitude

S2 Table. Two-sided t-test analysis on the extracted Optimism Indexes with zero. 